\title{
FOLLOW.ME - a system dedicated to patients with cognitive impairment
}

\author{
Athena Cristina Ribigan ${ }^{1,2}$, Raluca Stefania Badea ${ }^{1}, 0$ ctaviana Rusu', \\ Ovidiu Alexandru Bajenaru ${ }^{1,2}$, Florina Anca Antochi ${ }^{1}$ \\ ${ }^{1}$ Neurology Department, University Emergency Hospital, Bucharest, Romania \\ 2"Carol Davila" University of Medicine and Pharmacy, Bucharest, Romania
}

\begin{abstract}
Objectives. Neurocognitive impairment represents a frequent pathology among elderly people and not only, in the last decades the major focus of global health research being patient's safety. The aim of paper is to present a system designed for patients with all types of neurocognitive impairment that can improve the quality of life of not only the patients with major neurocognitive impairment but also of their relatives or informal caregivers.

Materials and methods. During October 2016 - February 2017 we used a multi-structured approach consisting in questionnaires, interviews, focus groups and co-creation sessions with the targeted people (neurologist, patients, caregivers) to identify and defi ne the characteristics of the system to be developed. We analysed the answers of 33 patients and informal caregivers and 27 neurologists from Romania, 29 informal caregivers and 52 formal caregivers from Belgium.

Results. Based on these data obtained, the technical partners participating in the project designed an application that could run on desktops, smartwatches, tablets and smartphones. The main features of this application are outdoor/indoor localization, guidance of the patient to a specific destination, assistance, notification, real time tracking and answering and it may help to quickly locate missing patients and to keep track of them in order to know if they leave or enter a given area (house, hospital, surroundings).

Conclusion. This monitoring system represents a new technology that patients, their relatives, caregivers and doctors can use in different environments to significantly increase the quality of life and also to avoid lifethreatening situations.
\end{abstract}

Keywords: elderly people, neurocognitive impairment, FOLLOW.ME, monitoring system

\section{INTRODUCTION}

Neurocognitive impairment is defined according to DSM V, published by the American Society of Psychiatry in 2013, as a clinical syndrome characterized by a significant decline compared with an anterior level of performance which affects one or more domains of cognition. This decline should be reported by the patient, observed by a relative, caregiver or a physician and documented by standardised neuropsychological tests or in the absence of such tests by other clinical methods that can be quantified. It should be mentioned that cognitive decline interferes with the independence of daily living and it is not related to delirium or other mental disorder (1).
The prevalence of neurocognitive impairment increases with age in an exponential manner. It doubles every five years after the age of 65 , being higher in female gender probably due to a higher life expectancy in this population, reaching 5-10\% in females with the age above 65 years old (2).

Neurodegenerative cognitive impairment is correlated with age. The prevalence is about $20 \%$ in persons aged more than 85 years old. Of all types of neurocognitive impairment, Alzheimer's disease (AD) is the most frequent one (3). Vascular neurocognitive impairment is the second most common type of cognitive impairment after AD (4).

It is estimated that 36 million people are affected by neurocognitive impairment worldwide but 
the number will almost double by 2030 , reaching 115 million by 2050 (5).

As this pathology is more and more frequent among elderly people and not only, in the last decades the major focus of global health research was patient's safety. The aim of this paper is to present a system designed for patients with all types of neurocognitive impairment that can improve the quality of life of not only the patients with major neurocognitive impairment but also of their relatives or informal caregivers.

\section{MATERIALS AND METHODS}

During the user needs and requirements analysis, the scientific methodology concerning data collection had a significant role. In this stage it has been used a multi-structured approach consisting in: questionnaires, interviews with the targeted people (neurologists, patients, informal caregivers) and focus groups. The stage of collecting and analyzing user requirements is based on relevant information and data collected from the Neurology Department of the University Emergency Hospital Bucharest and from specialized centers for elderly care of Belgium - LiCaLab vzw Belgium, Wit-Gele Kruis Limburg (WGKL) vzw Belgium. We analyzed the answers of 33 patients and informal caregivers and 27 neurologists from Romania, 29 informal caregivers and 52 formal caregivers (home care nurses and care professionals) from Belgium.

In order to identify and define the characteristics of the system to be developed for patients with neurocognitive impairment we created two types of questionnaires: one dedicated to medical staff and one dedicated to patients and informal caregivers.

WGKL developed a questionnaire for formal caregivers based on the questionnaire developed for neurologists but with few extra questions that were appropriate for professionals taking care of patients at home or in a residential care center.

This analysis was conducted between October 2016 - February 2017 with the aim to receive feedback regarding Follow.Me system.

The patients were included in the study only after the diagnosis of neurocognitive impairment any type was certain. Patients who were bedridden, with severe language impairment, severe visual or hearing problems and who did not signed the in- formed consent were excluded. The questionnaires comprised 28 different questions. When the patient was not able to respond to the survey a relative or an informal caregiver was asked to respond to the questions.

\section{RESULTS}

Of all the patients $55 \%$ presented suggestive symptoms for neurocognitive impairment for 1-5 years while only $21 \%$ of patients enrolled in the study had symptoms for about 5-10 years. The most frequent comorbidities among the study group were: hypertension $(79 \%)$, other cardiovascular diseases (52\%), depression (40\%), incontinence $(40 \%)$ and about one third had bone or joint disease.

One of the functionality that was considered useful by $91 \%$ of the patients was the alarming one which could remind them when to administer the medication.

Most of patients interviewed had an active life, $76 \%$ of patients being able to perform activities outside the house. The patients often had difficulties in performing daily living activities like driving the car (42\%) and cycling (40\%) but they still could perform shopping (70\%), periodic medical follow-up (55\%) or social activities (55\%). Only 6 of 33 patients performed activities only at home. More than $60 \%$ of the patients left the house more than once daily and most of them were able to walk on a distance of up to 1 kilometer. The favorite means of transportation were the personal automobile (patients being accompanied by e relative/caregiver) or public transportation. It is to be mentioned that $88 \%$ of the patients got support from a family member.

Nearly half of the interviewed patients and caregivers were familiar with the use of the digital technologies like computer, laptop, tablet or smartphone but they used the mobile phone only for calling and texting and not for GPS guiding the route to a destination although they considered it very useful when going outdoors.

The persons enrolled in the study thought that the most appropriate person to track the patients was a family member (91\%) and only less than $10 \%$ declared that other person should be in charge of this responsibility, the most important qualities 
of the tracking system being localization, detection of abnormal behavior and alarming in case of emergency.

The most important incidents that can trigger an alarm were: irregular heart rate $(73 \%)$, detection of long periods of inactivity (61\%), going out of a predefined area $(55 \%)$ but the patients/family or caregivers $(61 \%)$ would also prefer to have the opportunity to manually activate an alarm by tapping a special icon.

$85 \%$ of the family members and/or caregivers would prefer that the application ran on a smartphone and only $10 \%$ considered the smartwatch as an option.

The preferred way to be announced or cautioned of an event was by a sound (67\%). They also considered that the application could be improved by adding the phone number of the contact person (64\%), medical data of the patient, a memento for medication (48\%) or direction to the next hospital.

The responses of the patient/caregivers from other medical centers considered end-user of the this AAL project were in accordance with those presented above.

Also, the neurologists that participated in the survey considered that these features are very useful and would improve the quality of life of these patients and their families.

Based on these data mentioned earlier the technical partners participating in the project designed an application that could run on desktops, smatwatches, tablets and smartphones with all the functionalities described earlier (see the images below).

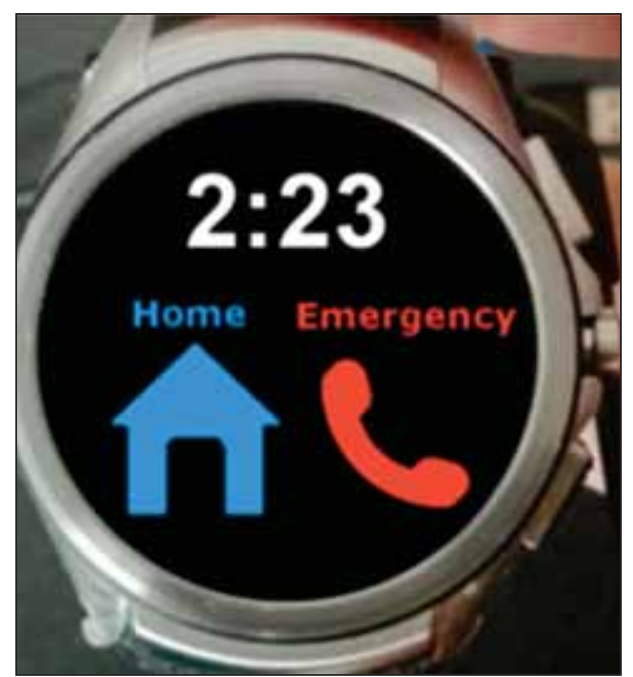

FIGURE 1. The main screen of the smartwatch.

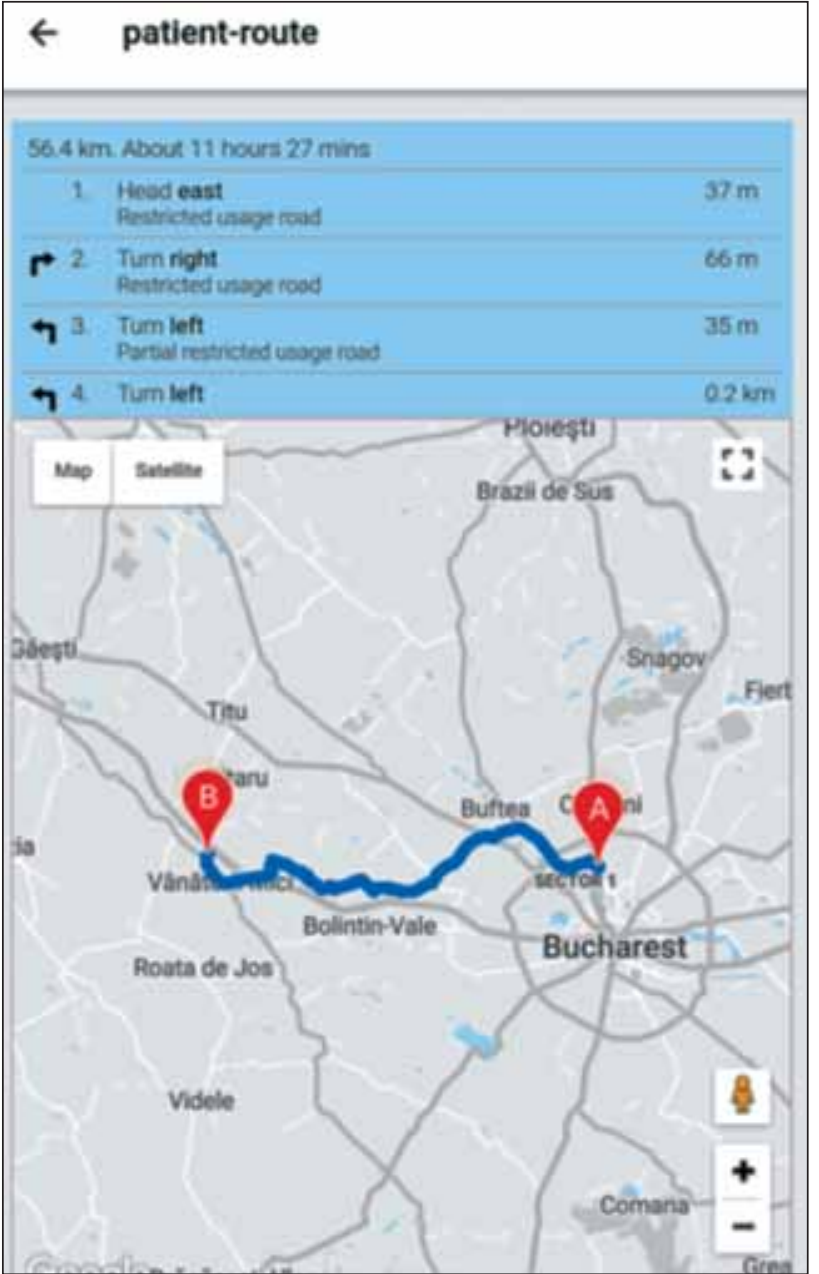

FIGURE 2. The route of the patient and guidance to destination (by a family member/caregiver) - mobile application designed for family members/caregivers/ doctors.

\section{CONCLUSIONS}

As people with neurocognitive impairment progressively limit their activities over years due to security concerns we created a monitoring system designed for those patients. This application will run on an independent smartwatch designed for patients and will provide guidance in case of disorientation, will monitor the patient's location, travel speed, periods of inactivity, heart rate both indoor and outdoor. In the same time the system will function on a smartphone/desktop designed for caregivers/doctors offering the same information.

This monitoring system represents a new technology that patients with neurocognitive impairment or other medical conditions, their relatives, caregivers and doctors can use in different environments to significantly increase the quality of life and also to avoid life-threatening situations. 


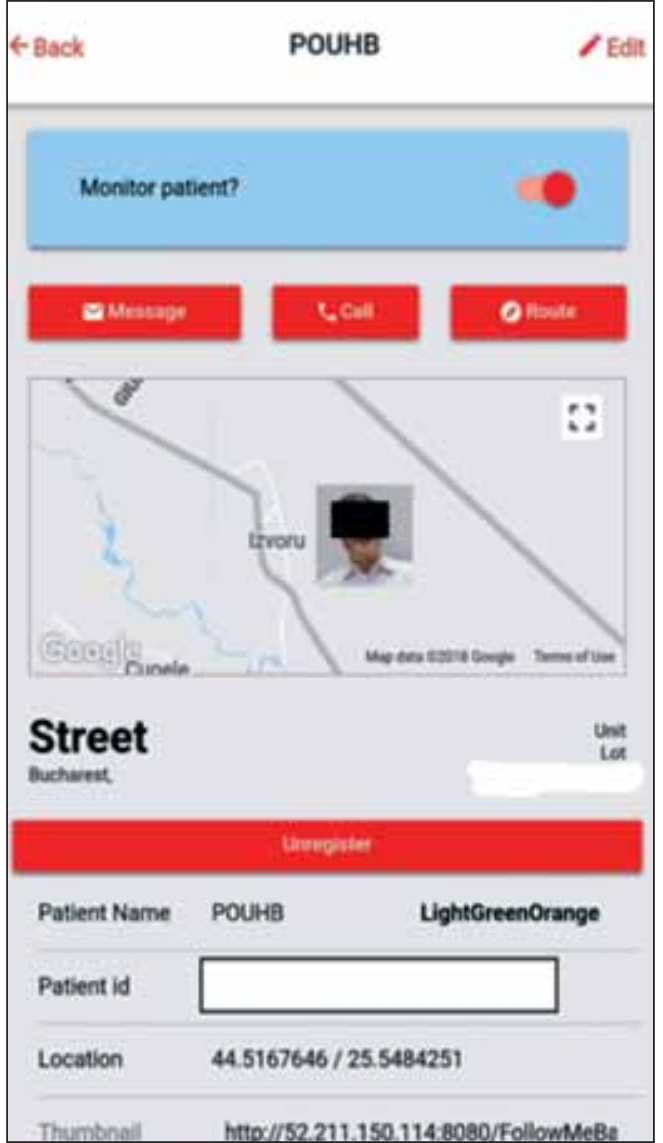

FIGURE 3. Information about one patient (possibility to insert pictures and written details). One caregiver/doctor may see details of more than one patient. It may be observed that one can call, text, see the route and guide the patient.

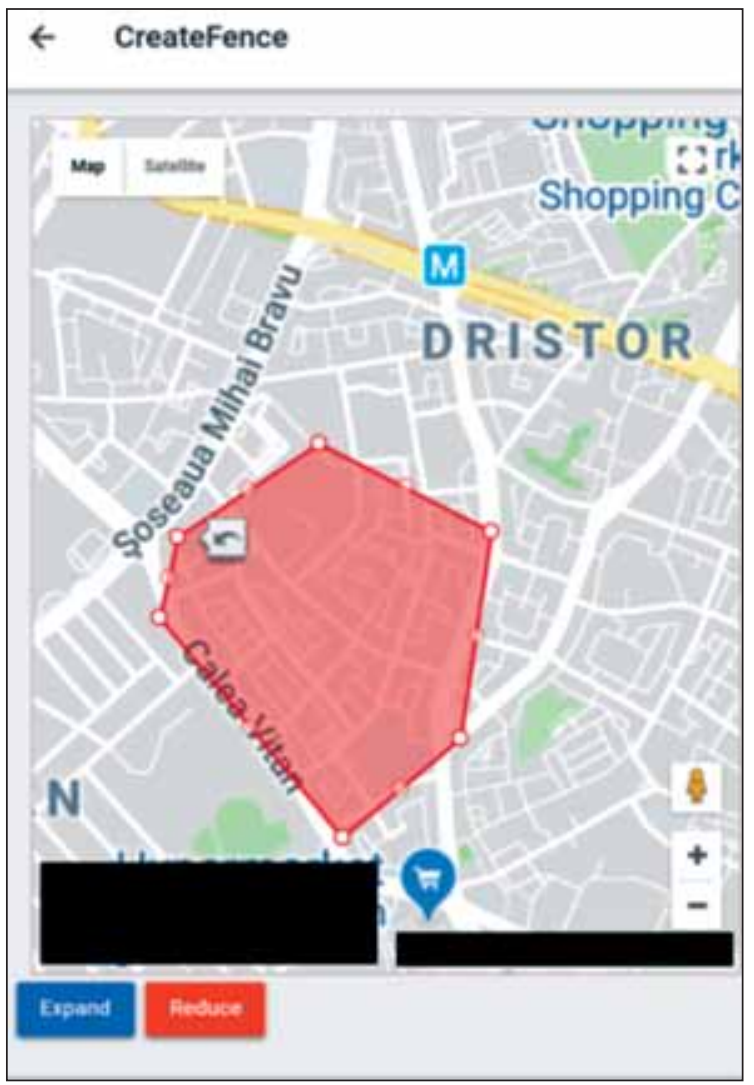

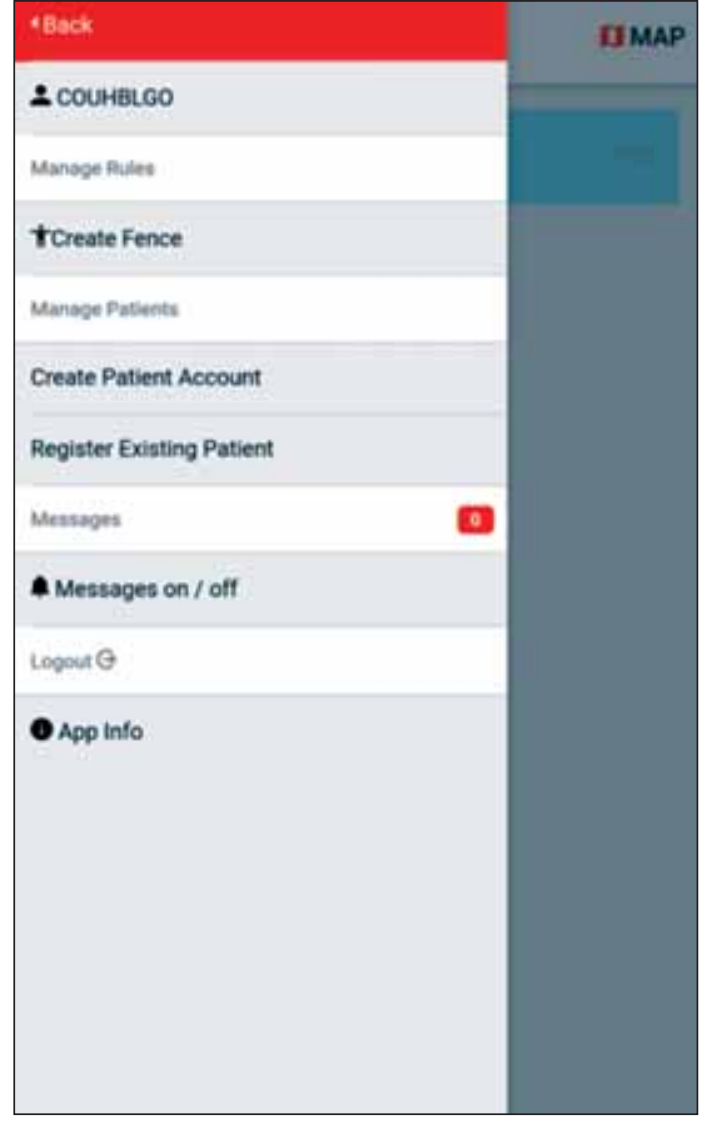

FIGURE 4. Editing information for one patient. One can create rules regarding the perimeter that patient is allowed to walk in (an alarm will be sent when the patient exits this area), the speed, heart rate. A new account can be created so more patients can be followed. 


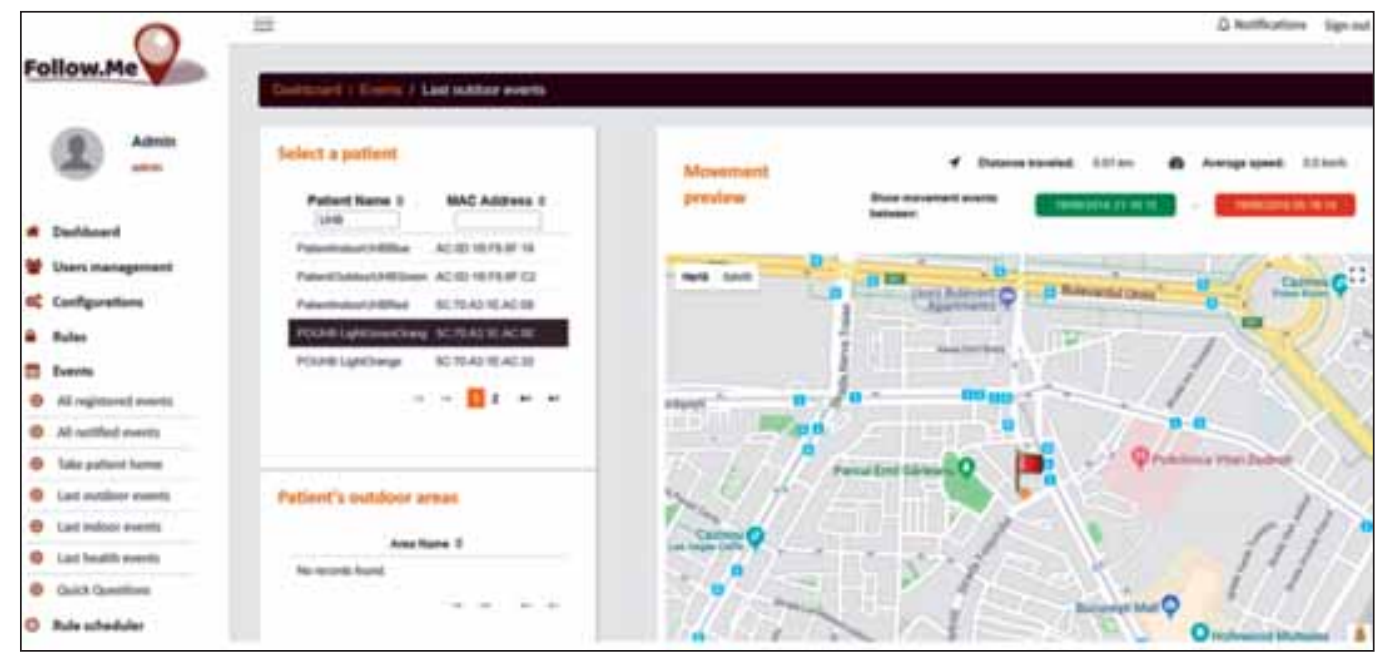

FIGURE 6. The desktop version of the application where all the setting could be performed. One can see the details about the patient's behavior (periods of inactivity, travel speed, heart rate) indoor (at home, hospital or other facility) or outdoor (yard, neighborhood, predefined area)

Conflict of interest: none declared Financial support: none declared

\section{REFERENCES}

1. American Psychiatric AAPA, Force DSMT. Diagnostic and statistical manual of mental disorders: DSM-5. Washington, D.C.: American Psychiatric Association. 2013.

2. Hugo J., Ganguli M. Dementia and cognitive impairment: epidemiology, diagnosis, and treatment. Clin Geriatr Med. 2014; 30(3):421-42.

3. Scott K.R., Barrett A.M. Dementia syndromes: evaluation and treatment. Expert Rev Neurother. 2007; 7(4):407-22.

4. Gorelick P.B., Scuteri A., Black S.E., Decarli C., Greenberg S.M., ladecola C. et al. Vascular contributions to cognitive impairment and dementia: a statement for healthcare professionals from the american heart association/american stroke association. Stroke. 2011; 42(9):2672-713.

5. Wortmann M. Dementia: a global health priority - highlights from an ADI and World Health Organization report. Alzheimers Res Ther. 2012; 4(5):40. 